\title{
Toward "green" mechanical simulations in materials science
}

\section{Hyper-reduction of a polycrystal plasticity model}

\author{
David Ryckelynck - Djamel Missoum Benziane \\ Andrey Musienko - Georges Cailletaud \\ MINES ParisTech, Centre des matériaux, CNRS UMR 7633 \\ BP 87, F-91003 Evry cedex \\ david.ryckelynck@mines-paristech.fr
}

\begin{abstract}
Because of the developpement of materials science, there is a need to reduce the computational complexity of mechanical models. This paper aims to show that the Hyper Reduction method enables to reduce computational resources used for numerical simulations. Large mechanical models involving distributed nonlinearities require parallel computers to solve the governing equations related to these models. The proposed Hyper Reduction of such models provides reduced governing equations that enable simulations on a single-processor computer. This is achieved by using a reduced-basis and a selection of equilibrium equations of the detailed model. The use of a single processor during less time enables to save an amazing amount of the electrical energy during the numerical simulation.

RÉSUMÉ. Du fait du développement des simulations numériques dans le domaine de la mécanique des matériaux, le besoin de méthodes pour une utilisation raisonnée des moyens de calcul se fait de plus en plus pressant. En effet, les connaissances acquises en science des matériaux et la disponibilité de moyens de calcul important facilitent la mise en auvre de modèles mécaniques non linéaires de très grande taille. Cet article a pour but de montrer que la méthode d'Hyper Réduction permet de réduire les ressources matérielles nécessaires afin de réaliser certaines simulations, pour des modèles extrêmement complexes validés expérimentalement. L'énergie électrique consommée pour réaliser la simulation est ainsi réduite fortement. Bien que la complexité du modèle hyper réduit soit moins grande que celle du modèle mécanique de référence, ce modèle conserve l'ensemble des paramètres physiques du modèle de référence.

KEYWORDS: truncated integration, POD, surrogate model, Petrov-Galerkin formulation.

MOTS-CLÉS : intégration tronquée, modèle d'ordre réduit, modèle de substitution, formulation de Petrov-Galerkin.
\end{abstract}

DOI:10.3166/EJCM.19.365-388 @ 2010 Lavoisier, Paris 


\section{Introduction}

Computational methods reducing the need of large computational resources have been already proposed in the literature. In case of linear models, the modal superposition method, the Guyan condensation method or the Craig-Bampton method (Craig et al., 1968) provide small size matrices establishing the coupled terms linking the reduced state variables of the models. These matrices can be easily transfered from a supercomputer to a smaller computer to perform reduced simulations. But in case of models involving distributed nonlinearities the solution of nonlinear local equations remains necessary when using reduced-bases. The complexity of these equations is not affected by the reduced-basis approximation. As shown in (Ryckelynck, 2009), the purpose of Hyper Reduction is to overcome this difficulty. Various model reduction methods are available for nonlinear models. From a theoretical point of view, the Hyper Reduction method can be applied on Proper Orthogonal Decomposition (POD) (Lorenz, 1956) or Radial Loading Decomposition (RLD) generated by the LATIN method (Ladevèze, 1985). A first attempt to reduce a mechanical model in the framework of materials science using POD basis has been proposed by S. Ganapathysubramanian and $\mathrm{N}$. Zabaras. These authors had applied the POD reduction method to a polycrystal plasticity problem. The usefulness of POD basis for sensitivity analyses related to this kind of model, in the framework of an optimization problem, have been stated in (Ganapathysubramanian et al., 2004). The present paper aims to show that the Hyper Reduction method enables to reduce the computational resources needed to use POD-bases generated by the snapshot POD method (Sirovich, 1987). It is the continuation of previous works related to the three-dimensional finite element simulation of a polycrystalline copper specimen (Musienko et al., 2007). The detailed model is a single crystal plasticity model. It represents the elasto-plastic behavior for each of the approximately 200 grains involved in the specimen.

A detailed model is converted into a Reduced-Order Model (ROM) supposing that the state variables belong to a restricted functional subspace. Therefore the number of independent state variables (the order of the model) is reduced. The purpose of a model reduction method is to build a basis of this subspace. The POD model reduction method has been used in a wide range of disciplines including signal analysis and simulation in fluid mechanics or in oceanography (Holmes et al., 1998). This method comes from the Karhunen-Loève expansion (Karhunen, 1946; Loève, 1963) developed for statistical analyses. In our knowledge, the first mechanical models using POD bases were proposed in (Lorenz, 1956) for weather prediction using experimental data. A POD basis is a basis of the state subspace spanned by forecasted states possibly related to different simulations of the response of the system. The development of large Finite Element models increases the need of low order models created by model reduction methods. The availability of ROMs can greatly facilitate the solution of optimization problems. The efficiency of POD bases was shown for optimization problems in fluid mechanics (Daescu et al., 2007; Lieu et al., 2006), in structural dynamics (Khalila et al., 2007), in materials science (Ganapathysubramanian et $a l ., 2004)$ and in thermal science (Balima et al., 2006). 
The aim of this paper is not to improve the creation of a POD basis. We improve the use of a given POD basis in the framework of highly complex mechanical models in good agreement with experimental observations. Because the complexity of the local computations is not dependent on the order of the model, the reduced approximation has no effect on the computational effort needed to estimate the internal variables. This limits the efficiency of the reduced approximation. The main idea to overcome this difficulty is to choose convenient equilibrium conditions to compute the reduced state variables. Obviously the number of reduced governing equations must be equal to the number of reduced state variables in order to get a well-posed problem. But it is not necessary to build a formulation based on all the equilibrium conditions of the detailed model to define the reduced governing equations. Therefore we propose a convenient vector space to define the test functions of the virtual work principle. The formulation of the constitutive equations remains the same. The hyper-reduction method lower the complexity of models in the sense that symmetrical models are less complex than $3 \mathrm{~d}$ models. The global complexity is changed.

The virtual work is an extensive variable. Let us consider a mechanical system split in two complementary parts $\Omega_{\Pi}$ and $\bar{\Omega}_{\Pi}$. Therefore, the virtual work of the internal forces related to this system is the sum of the contribution of each part. The Hyper Reduction method proposed in (Ryckelynck, 2005; Ryckelynck, 2009) introduces a Reduced Integration Domain (RID) $\Omega_{\Pi}$ and a space of truncated test functions in order to generate the surrogate model. The Hyper Reduced Order Model (HROM) can be created using an adaptive procedure as proposed in (Ryckelynck, 2005; Ryckelynck et al., 2010). The truncated test functions are such that the contribution of $\bar{\Omega}_{\Pi}$ to the virtual work of internal forces is equal to zero. The truncated test functions are admissible test functions having their support retricted to the RID. The size of the space of truncated test functions remains equal to the size of the ROM, but the local equations of the surrogate model are resticted to the RID. The shape functions related to the reduced-basis having a global support, the knowledge of the reduced state variables enables to forecast the state of all the mechanical system even if these variables have been computed using a RID. Therefore, when using a domain decomposition formulation (Farhat et al., 1994), the Hyper Reduction enables to use a reduced-order model over only one subdomain to forecast the state of the entire domain. Hence the computational resources needed to compute the reduced state variables can be reduced. Moreover, the reduced approximation being continuous overall the domain, the continuity of the displacement is naturaly enforced by this approximation. Its not necessary to introduce Lagrange multipliers into the formulation of the equilibrium equations to enforce continuity constrains between subdomains.

No local computation being made outside the RID, no internal variable are forecast outside the RID when solving the equilibrium conditions related to the surrogate model. As proposed in (Ryckelynck, 2009), a POD basis devoted to internal variables is generated simultaneously with the POD basis related to the displacements. This added basis enables to extrapolate the computed internal variables form the RID to the full mechanical system. The reader must be aware that the Hyper Reduction method amplifies the errors due to the reduced approximation of the displacements. 
The paper is organised in the following manner. Section 2 introduces the formulation of the mechanical problem involving internal variables. The Hyper Reduction method is detailed in Section 3. A simple numerical experiment of the Hyper Reduction method is proposed in Section 4. Section 5 reports the numerical results elucidating the usefulness of the proposed method for simplified sensitivity analysis. Section 5 is the conclusion of this paper.

\section{Problem statement}

\subsection{Formulation of the continuous model}

The continuous model of concern is a parametrized mechanical model. We denote $\{p\}$ the column of the model parameters. These parameters can be related to design choices, material coefficients or boundary conditions. We consider a series of mechanical problems related to a series of parameter values $\left(\{p\}_{\alpha}\right)_{\alpha=1 \ldots N}$. The detailed model is described using the finite strain formalism. The reference configuration is denoted $\Omega^{0}$. It can be the domain eather at time instant $t=0$ (total Lagrangian formulation) or at time instant $t$ (update Lagrangian formulation). At time instant $t$, the continuous medium is occupying a domain $\Omega$. The nonlinear system is analyzed over a time interval $] 0, T]$. The displacement field at time $t$ is defined on $\Omega^{0}$ and it is denoted by $\mathbf{u}\left(\mathbf{X}, t,\{p\}_{\alpha}\right)$. $\mathbf{X}$ is denoting the initial position of a material point in $\Omega^{0}$. The second Piola-Kirchhoff stress tensor $\mathbf{S}$ is a nonlinear function of the deformation gradient history depending on the parameters $\{p\}_{\alpha}$ :

$$
\mathbf{S}=\mathbf{\Sigma}\left(\mathbf{F}_{\tau}, \tau \leq t ;\{p\}_{\alpha}\right)
$$

where $\boldsymbol{\Sigma}$ is a formal operator that must be defined by constitutive equations and $\mathbf{F}_{\tau}$ is the deformation gradient at time instant $\tau$. We observe that the following general relations hold:

$$
\begin{aligned}
F_{i j} & =\delta_{i j}+\frac{\partial u_{i}}{\partial X_{j}} \\
\boldsymbol{\sigma} & =\frac{1}{\operatorname{det}(\mathbf{F})} \mathbf{F S F}^{T}
\end{aligned}
$$

where $\boldsymbol{\sigma}$ is the Cauchy stress tensor and $\delta_{i j}$ is the Kronecker delta.

The boundary $\partial \Omega^{0}$ of $\Omega^{0}$ is denoted by $\partial_{U} \Omega^{0} \cup \partial_{f} \Omega^{0}$. On $\partial_{U} \Omega^{0}$, there is the Dirichlet condition $\mathbf{u}\left(., t,\{p\}_{\alpha}\right)=\mathbf{u}_{c}\left(., t,\{p\}_{\alpha}\right)$ for all $t$, where $\mathbf{u}_{c}$ is a given parametrized displacement field. On $\partial_{f} \Omega^{0}$, there is a given force field $\mathbf{f}\left(., t,\{p\}_{\alpha}\right)$ depending on both time $t$ and model parameters. The displacement field belongs to an affine function space $\mathcal{U}$ defined by:

$$
\begin{array}{r}
\mathcal{U}\left(\{p\}_{\alpha}\right)=\left\{\mathbf{u}\left(., t,\{p\}_{\alpha}\right) \in H^{1}\left(\Omega^{0}\right) \mid \mathbf{u}\left(\mathbf{X}, t,\{p\}_{\alpha}\right)=\mathbf{u}_{c}\left(\mathbf{X}, t,\{p\}_{\alpha}\right)\right. \\
\left.\forall \mathbf{X} \in \partial_{U} \Omega^{0}\right\}
\end{array}
$$


The vector space associated to $\mathcal{U}\left(\{p\}_{\alpha}\right)$ is denoted $\mathcal{V}$. It does not depend on any parameter:

$$
\mathcal{V}=\left\{\mathbf{u}\left(., t,\{p\}_{\alpha}\right) \in H^{1}\left(\Omega^{0}\right) \mid \mathbf{u}\left(\mathbf{X}, t,\{p\}_{\alpha}\right)=0 \quad \forall \mathbf{X} \in \partial_{U} \Omega^{0}\right\}
$$

The statement of the mechanical problem is the following. We want to find an estimation of the displacement field $\mathbf{u} \in \mathcal{U}\left(\{p\}_{\alpha}\right)$ defined by the constitutive equations and the principle of virtual work:

$$
\begin{array}{r}
\int_{\Omega^{0}} \boldsymbol{\varepsilon}\left(\mathbf{u}^{*}, \mathbf{u}\right): \boldsymbol{\Sigma}\left(\mathbf{F}\left(\mathbf{u}_{\tau}\right), \tau \leq t,\{p\}_{\alpha}\right) d \Omega^{0} \\
-\int_{\partial_{f} \Omega^{0}} \mathbf{u}^{*} \cdot \mathbf{f}\left(\mathbf{X}, t,\{p\}_{\alpha}\right) d \Gamma^{0}=0 \\
\forall \mathbf{u}^{*} \in \mathcal{V}
\end{array}
$$

where $\mathbf{u}^{*}$ is a test function and $\varepsilon$ is a linear function of $\mathbf{u}^{*}$ such that :

$$
\varepsilon_{i j}\left(\mathbf{u}^{*}, \mathbf{u}\right)=\frac{1}{2}\left(\frac{\partial u_{i}^{*}}{\partial X_{j}}+\frac{\partial u_{j}^{*}}{\partial X_{i}}+\sum_{k=1}^{k=3}\left(\frac{\partial u_{k}^{*}}{\partial X_{i}} \frac{\partial u_{k}}{\partial X_{j}}+\frac{\partial u_{k}}{\partial X_{i}} \frac{\partial u_{k}^{*}}{\partial X_{j}}\right)\right)
$$

According to the framework of the irreversible thermodynamic processes, a constitutive law can be defined by a choice of: internal variables $\mathbf{z}$, a free energy $w\left(\varepsilon, \mathbf{z},\{p\}_{\alpha}\right)$ and a pseudo potential of dissipation $\varphi^{*}$ (Germain et al., 1983). Some conjugated variables $\mathbf{Z}$ are associated to the internal variables $\mathbf{z}$ using the definition of the dissipation. The conjugated variables and the internal variables are connected by the following equation of state:

$$
\mathbf{Z}=-\frac{\partial w}{\partial \mathbf{z}}
$$

Complementary constitutive equations can be proposed without introducing a pseudo potential of dissipation, provided that the Clausius Duhem Inequality is fulfilled (the rate of entropy production must not be negative):

$$
\dot{\mathbf{z}}=\mathbf{B}\left(\mathbf{Z},\{p\}_{\alpha}\right)
$$

In case of standard formulation of the constitutive equations, the complementary constitutive equations are deduced from the pseudo potential of dissipation $\varphi^{*}\left(\mathbf{Z},\{p\}_{\alpha}\right)$ such that:

$$
\mathbf{B}\left(\mathbf{Z},\{p\}_{\alpha}\right)=\frac{\partial \varphi^{*}}{\partial \mathbf{Z}}
$$


The initial state of the material is defined by a given initial condition:

$$
\mathbf{z}_{\mid t=0}=\mathbf{z}_{\text {ini }}
$$

Examples of elastoplastic or elastoviscoplastic constitutive models can be found in (Lemaitre et al., 1990; Sansour et al., 1997).The Pilola-Kirchhoff stress $\mathbf{S}$ being one of the conjugate variables, the set of equations Equation [8] to Equation [11] defines the operator $\boldsymbol{\Sigma}$. These equations are local equations: the strain tensor at the point $X^{\prime}$ is not a variable of the constitutive equations related to the point $X \neq X^{\prime}$. The detailed equations of the constitutive law related to polycrystalline copper specimen are given in Section 5.

The main objective of the model reduction methods is to replace $\mathcal{U}\left(\{p\}_{\alpha}\right)$ and $\mathcal{V}$ by small dimensional functional subspaces in order to approximate $\mathbf{u}\left(., t,\{p\}_{\alpha}\right)$ using previous results of simulations.

\subsection{The reference model}

Using the Finite Element method (Zienkiewicz, 2000), a backward Euler method and the Newton-Raphson algorithm, one can forecast different states of the system at different time instants. The purpose of is paper is not to explain how to get these states. Details on the finite element simulation of crystal plasticity models can be found in (Forest et al., 1999; Tugcu et al., 2004) for instance. This section aims to recall some properties of the states forecast by a Finite Element model because it is the detailed model to reduce. Considering large dimensional finite element problems, a domain decomposition and a FETI solver enable to perform parallel computations (Farhat $e t$ al., 1994). An example of such a method applied to a polycrystal model can be found in (Feyel et al., 1997). The domain $\Omega^{0}$ is split into $\omega$ subdomains:

$$
\Omega^{0}=\cup_{\eta=1}^{\eta=\omega} \widehat{\Omega}_{\eta}^{0}
$$

Using a FETI solver, we can distinguish two Finite Element models. One of these models defines a continuous kinematic description over $\Omega^{0}$. Let us denote $\mathcal{U}_{h}$ and $\mathcal{V}_{h}$ the functional affine space and the vector space related to this model. The other finite element model does not impose the continity of the displacement between the subdomains. The displacement continuity is enforced by Lagrange multipliers. The related functional affine subspace is $\widehat{\mathcal{U}}_{h}$. 
The discontinuous Finite Element model involves $\widehat{n}$ degrees of freedom $\left(\widehat{q}_{j}(t)\right)_{j=1 . . \widehat{n}}$ such that:

$$
\begin{gathered}
\widehat{\mathcal{U}}_{h}=\left\{\widehat{\mathbf{u}}_{h} \in \mathcal{V} \mid \exists\{\widehat{q}\} \in \Re^{\widehat{n}},\right. \\
\widehat{\mathbf{u}}_{h}\left(\mathbf{X}, t,\{p\}_{\alpha}\right)=\sum_{j=1}^{j=\widehat{n}} \widehat{\mathbf{N}}_{j}(\mathbf{X}) \widehat{q}_{j}\left(t,\{p\}_{\alpha}\right)+\sum_{j=1}^{j=n_{c}} \widetilde{\mathbf{N}}_{j}(\mathbf{X}) g_{j}\left(t,\{p\}_{\alpha}\right) \\
\left.\left.\left.\forall \mathbf{X} \in \Omega^{0} \quad \forall t \in\right] 0, T\right]\right\}
\end{gathered}
$$

The shape functions $\left(\widetilde{\mathbf{N}}_{j}\right)_{j=1, \ldots n_{c}}$ are connected to the nodes belonging to $\partial_{U} \Omega^{0}$. The coefficient $g_{j}$ are given displacement related to $\mathbf{u}_{c}$. Therefore, the given part of Finite Element displacement field is $\mathbf{u}_{c h}$ such that:

$$
\mathbf{u}_{c h}\left(\mathbf{X}, t,\{p\}_{\alpha}\right)=\sum_{j=1}^{j=n_{c}} \widetilde{\mathbf{N}}_{j}(\mathbf{X}) g_{j}\left(t,\{p\}_{\alpha}\right)
$$

Let us denote $\widehat{\mathcal{V}}_{h}$ the vector subspace span by the basis $\left(\widehat{\mathbf{N}}_{j}\right)_{j=1, \ldots .}$ :

$$
\widehat{\mathcal{V}}_{h}=\operatorname{span}\left\{\widehat{\mathbf{N}}_{1}, \ldots, \widehat{\mathbf{N}}_{\widehat{n}}\right\}
$$

In this paragraph we give few indications about the FETI method. We refer the reader to (Farhat et al., 1994) for more details about this method. In spite of the fact that approximate continuous displacement field are obtained using itermediate discontinuous fields, the way it is performed has no influence on the implementation of the Hyper Reduction method. To enforce the displacement continuity the following equation must be fulfilled:

$$
[B] \cdot\{\widehat{q}\}=0
$$

Moreover, the domain beeing split into subdomains, some rigid modes can appear. These modes span the null space of the stiffness matrix related to the whole split domain. The matrix containing the rigid modes is denoted $\left[Q_{r}\right]$. The nodal displacement are written as a sum of the contribution of the rigid modes plus a contribution $\left\{\widehat{q}_{K}\right\}$ which is orthogonal to the null space of the stiffness matrix such that:

$$
\{\widehat{q}\}=\left\{\widehat{q}_{K}\right\}+\left[Q_{r}\right] \cdot\left\{h_{r}\right\} \quad \text { with } \quad\left[Q_{r}\right]^{T} \cdot\left\{\widehat{q}_{K}\right\}=0
$$

The continuity condition can be enforced using Lagrange multipliers $\{\lambda\}$ if the following equilibrium condition is fulfilled:

$$
\left[Q_{r}\right]^{T} \cdot\left(\left\{F_{e}\right\}-[B]^{T} \cdot\{\lambda\}\right)=0
$$

where $\left\{F_{e}\right\}$ is the vector related to the external forces applied to the domain. Then, a formal static condensation of $\left\{\widehat{q}_{K}\right\}$ enables to introduce a global system of equations 
related to the unknowns $\left(\left\{h_{r}\right\},\{\lambda\}\right)$. To avoid the implementation of this expensive condensation, a conjugate gradient algorithm is introduce to solve the global system of equations. Each step of this iterative algorithm includes a prediction of $\left\{\widehat{q}_{K}\right\}$. Thanks to the domain decomposition this prediction is performed subdomain by subdomain, since it is related to uncoupled mechanical problems.

In case of matching meshes between subdomains, the definition of a continuous Finite Element model is straighforward using a selection matrix $[P]$. This matrix is a rectangular matrix such that $\overline{\{q\}} \in \Re^{\widehat{n}}$, with $\overline{\{q\}}=[P]^{T} \cdot[P] \cdot \widehat{\{q\}}$, provides a continuous field $\mathbf{u}_{h}\left(\mathbf{X}, t,\{p\}_{\alpha}\right) \in \mathcal{V}_{h}$ such that:

$$
\mathbf{u}_{h}\left(\mathbf{X}, t,\{p\}_{\alpha}\right)=\sum_{j=1}^{j=\widehat{n}} \widehat{\mathbf{N}}_{j}(\mathbf{X}) \bar{q}_{j}
$$

The number of rows of the selection matrix $[P]$ is equal to the number of degrees of freedom of the continuous Finite Element model. Various definitions of $[P]$ are available to obtain a projection operator $[P]^{T} .[P]$ such that a continuous field is not modified:

$$
[P]^{T} \cdot[P] \cdot \widehat{\{q\}}=[P]^{T} \cdot[P] \cdot[P]^{T} \cdot[P] \cdot \widehat{\{q\}} \quad \forall \widehat{\{q\}} \in \Re^{\widehat{n}}
$$

The matrix $[P]$ enables to define the shape functions of the continuous Finite Element model $\left(\mathbf{N}_{j}\right)_{j=1, \ldots n}$ such that:

$$
\begin{aligned}
& \mathbf{N}_{j}(\mathbf{X})=\sum_{k=1}^{k=\widehat{n}} \widehat{\mathbf{N}}_{k}(\mathbf{X}) P_{j k} \quad \forall \mathbf{X} \in \Omega^{0} \\
& \mathcal{V}_{h}=\operatorname{span}\left\{\mathbf{N}_{1}, \ldots, \mathbf{N}_{n}\right\}
\end{aligned}
$$

Because the continuity of the displacement fields is enforced by the FETI solver, the various displacement field forecast at the end of each time increment are assumed to be continuous. They belong to the functional space $\mathcal{U}_{h}$. Removing the imposed part $\mathbf{u}_{c h}$ of the displacement fields, we are able to obtain a series of continuous displacements $\mathbf{U}_{i} \in \mathcal{V}_{h}$ for $i=1 \ldots m$. Therefore it is not necessary to introduce Lagrange multipliers to enforce continuity conditions in equilibrium equation in order to introduce the ROM. The classical formulation, Equation [7], is sufficient even if the domain is split into subdomains.

The displacement fields $\mathbf{U}_{i}$ can be related to various parameter values $\{p\}_{\alpha}$ and various time instants.

\subsection{The reduced approximation}

The purpose of the snapshot POD is to use the list of the forecasted displacement fields $\left(\mathbf{U}_{i}\right)_{i=1 \ldots m}$ to define a reduced basis of the subspace span by these fields. Let 
us denote $\left(\psi_{k}\right)_{k=1 \ldots \widehat{m}}$ the vectors of the reduced basis (in practice $\widehat{m}<n$ ). Let us denote $\mathcal{V}_{P O D}$ the vector space related to the ROM:

$$
\mathcal{V}_{P O D}=\operatorname{span}\left\{\boldsymbol{\psi}_{1}, \ldots, \boldsymbol{\psi}_{\widehat{m}}\right\}
$$

Later, new state estimations related to modifications of the model can be computed using the following reduced approximation:

$$
\begin{array}{r}
\mathbf{u}_{P O D}\left(\mathbf{X}, t,\{p\}_{\alpha}\right)=\sum_{k=1}^{k=\widehat{m}} \psi_{k}(\mathbf{X}) a_{k}\left(t,\{p\}_{\alpha}\right)+\mathbf{u}_{c h}\left(\mathbf{X}, t,\{p\}_{\alpha}\right) \\
\left.\left.\forall \mathbf{X} \in \Omega^{0} \quad \forall t \in\right] 0, T\right]
\end{array}
$$

The factors $\left(a_{k}\right)_{k=1 \ldots \widehat{m}}$ are the reduced state variables of the ROM. The affine space related to the reduced basis approximation in Equation [24] is denoted $\mathcal{U}_{P O D}\left(\{p\}_{\alpha}\right)$ :

$$
\mathcal{U}_{P O D}\left(\{p\}_{\alpha}\right)=\left\{\mathbf{u}_{P O D} \in \mathcal{U}\left(\{p\}_{\alpha}\right) \mid \mathbf{u}_{P O D}-\mathbf{u}_{c h} \in \mathcal{V}_{P O D}\right\}
$$

The snapshot POD was proposed by L. Sirovich (Sirovich, 1987). The vector $\psi_{k}$ of the reduced basis are found inside the subspace spanned by the computed displacement fields such that:

$$
\boldsymbol{\psi}_{k}(\mathbf{X})=\sum_{i=1}^{i=m} \mathbf{U}_{i}(\mathbf{X}) b_{i k} \quad \forall \mathbf{X} \in \Omega^{0}
$$

where $b_{i k}$ must maximise the projection $\lambda_{k}$ of $\psi_{k}$ on all the snapshots, such that:

$$
\lambda_{k}=\frac{\sum_{j=1}^{j=m}\left(\int_{\Omega^{0}} \mathbf{U}_{j} \cdot \boldsymbol{\psi}_{k} d \Omega^{0}\right)^{2}}{\int_{\Omega^{0}}\left\|\boldsymbol{\psi}_{k}\right\|^{2} d \Omega^{0}}
$$

L. Sirovich proved in (Sirovich, 1987) that the matrix $[b]$ contains the eigenvectors of the covariance matrix $[M]$ such that:

$$
M_{i j}=\int_{\Omega^{0}} \mathbf{U}_{i} \cdot \mathbf{U}_{j} d \Omega^{0}
$$

$[M]$ being a symmetric and positive matrix of size $m$, the matrix $[b]$ contains $m$ eigenvectors. The $k^{t h}$ column of $[b]$ is related to the eigenvalue $\lambda_{k}$ such that $\lambda_{k}>\lambda_{k+1} \geq$ 0 . Some of the eigenvectors can have a negligible contribution to the approximation equation in Equation [24]. In practice, we remove these negligible contributions by a selection of the $\widehat{m}$ first columns of $[b]$ such that:

$$
\sum_{k=1}^{k=\widehat{m}} \lambda_{k} \geq(1-\epsilon) \sum_{k=1}^{k=m} \lambda_{k}
$$


where $\epsilon$ is a positive parameter of the snapshot POD. In practice we choose $\epsilon=$ $10^{-8}$. $\mathcal{V}_{P O D}$ being a subspace of $\mathcal{V}_{h}$, we can introduce a reduction matrix $[A]$ such that :

$$
\boldsymbol{\psi}_{k}(\mathbf{X})=\sum_{j=1}^{j=n} \mathbf{N}_{j}(\mathbf{X}) A_{j k} \quad \forall \mathbf{X} \in \Omega^{0}
$$

In case of domain decomposition for parallel computing, each displacement vector is split such that each subdomain $\widehat{\Omega}_{\eta}^{0}$ has its own contribution, $\mathbf{U}_{j \eta}$, to the global vectors.

$$
\mathbf{U}_{j}=\mathbf{U}_{j \eta} \quad \forall \mathbf{X} \in \widehat{\Omega}_{\eta}^{0}
$$

It turns out that the computation of the matrix $[M]$ is distributed over all the processors used for the parallel computation:

$$
M_{i j}=\sum_{\eta=1}^{\eta=\omega} \int_{\Omega_{\eta}^{0}} \mathbf{U}_{i \eta} \cdot \mathbf{U}_{j \eta} d \Omega^{0}
$$

Using a single program and multiple data approach, the eigenvector $\{b\}_{k}$ of the matrix $[M]$ are forecast on each processor. Therefore each processor as its own contribution to each global vector:

$$
\boldsymbol{\psi}_{k}(\mathbf{X})=\sum_{i=1}^{i=m} \mathbf{U}_{i \eta}(\mathbf{X}) b_{i k} \quad \forall \mathbf{X} \in \widehat{\Omega}_{\eta}^{0} \quad \forall \eta \quad \forall k=1, \ldots, \widehat{m}
$$

\subsection{The classical POD-Galerkin formulation of the reduced equilibrium equations}

The classical Galerkin formulation of the equilibrium equation of the Finite Element model is obtained by replacing $\mathcal{V}$ by $\mathcal{V}_{h}$ and $\mathcal{U}$ by $\mathcal{U}_{h}$ in Equation [7]. In a similar way, the Galerkin formulation of the reduced equilibrium condition is obtained by replacing $\mathcal{V}$ by $\mathcal{V}_{P O D}$ and $\mathcal{U}$ by $\mathcal{U}_{P O D}$ in Equation [7]. 
The formulation of the reduced order model is the following. Find the displacement field $\mathbf{u}_{P O D} \in \mathcal{U}_{P O D}\left(\{p\}_{\alpha}\right)$ defined by the constitutive equations and the principle of virtual work:

$$
\begin{array}{r}
\int_{\Omega^{0}} \varepsilon\left(\mathbf{u}_{P O D}^{*}, \mathbf{u}_{P O D}\right): \boldsymbol{\Sigma}\left(\mathbf{F}\left(\mathbf{u}_{P O D}\right), \tau \leq t,\{p\}_{\alpha}\right) d \Omega^{0} \\
-\int_{\partial_{f} \Omega^{0}} \mathbf{u}_{P O D}^{*} \cdot \mathbf{f}\left(\mathbf{X}, t,\{p\}_{\alpha}\right) d \Gamma^{0}=0 \\
\forall \mathbf{u}_{P O D}^{*} \in \mathcal{V}_{P O D}
\end{array}
$$

The quality of the ROM prediction can be checked using the residue $\{R\}$ of the Finite Element equilibrium condition such that:

$$
\begin{gathered}
R_{j}\left(\{q\}, t,\{p\}_{\alpha}\right)=\int_{\Omega^{0}} \varepsilon\left(\mathbf{N}_{j}, \mathbf{u}\right): \mathbf{\Sigma}\left(\mathbf{F}\left(\mathbf{u}_{\tau}\right), \tau \leq t,\{p\}_{\alpha}\right) d \Omega^{0} \\
-\int_{\partial_{f} \Omega^{0}} \mathbf{N}_{j} \cdot \mathbf{f}\left(\mathbf{X}, t,\{p\}_{\alpha}\right) d \Gamma^{0}
\end{gathered}
$$

with

$$
\mathbf{u}\left(\mathbf{X}, t,\{p\}_{\alpha}\right)=\sum_{i=1}^{i=n} \mathbf{N}_{i}(\mathbf{X}) q_{i}\left(t,\{p\}_{\alpha}\right)+\mathbf{u}_{c h}\left(\mathbf{X}, t,\{p\}_{\alpha}\right)
$$

A relative error can be defined as follows:

$$
\eta=\max _{t \in[0, T]} \frac{\left\|\{R\}\left([A] \cdot\{a\}, t,\{p\}_{\alpha}\right)\right\|}{\left\|\{R\}\left(0, t,\{p\}_{\alpha}\right)\right\|}
$$

$\varepsilon$ being a linear function of $\mathbf{u}^{*}$, the matrix form of the reduced equilibrium equation, Equation [36], is the following:

$$
[A]^{T} \cdot\{R\}\left([A] \cdot\{a\}, t,\{p\}_{\alpha}\right)=0
$$

\section{Formulation of the hyper-reduction method}

For both models, the detailed one and the reduced one, the nonlinear local constitutive equations have to be solved to estimate the stress field $\boldsymbol{\Sigma}\left(\mathbf{F}\left(\mathbf{u}_{\tau}\right), \tau \leq t,\{p\}_{\alpha}\right)$ at any point of the domain $\Omega^{0}$. The fact that $\mathbf{u}_{P O D}$ belongs to $\mathcal{U}_{P O D}\left(\{p\}_{\alpha}\right)$ has no effect on the complexity of the constitutive equations involved in Equation [36]. The Galerkin procedure does not modify the number of constitutive equations that must be solved to forecast $\mathbf{u}_{P O D} \in \mathcal{U}_{P O D}\left(\{p\}_{\alpha}\right)$. 
One way to reduce the number of constitutive equations is the creation of a Reduced Integration Domain (RID). The orthogonal condition, Equation [39], does not mean that all the residues of the equilibrium equations must be equal to zero. Using a RID, we do not include all the residues $R_{j}(j=1 \ldots n)$ into the formulation of the reduced governing equations. Let us split the domain $\Omega^{0}$ in two complementary parts $\Omega_{\Pi}^{0}$ and $\bar{\Omega}_{\Pi}^{0}$ :

$$
\Omega^{0}=\Omega_{\Pi}^{0} \oplus \bar{\Omega}_{\Pi}^{0}
$$

Introducing a subspace of truncated test functions, $\mathcal{V}_{\Pi}$, we can select few equilibrium equations of the detailed model:

$$
\begin{aligned}
\mathcal{V}_{\Pi}= & \left\{\mathbf{u}_{\Pi} \in \mathcal{V} \mid \exists\{q\} \in \Re^{n},\right. \\
& \left.\mathbf{u}_{\Pi}(\mathbf{X})=\sum_{j=1}^{j=n} \mathbf{N}_{j}(\mathbf{X}) \Lambda_{j j} q_{j} \quad \forall \mathbf{X} \in \Omega^{0}\right\}
\end{aligned}
$$

where $[\Lambda]$ is a diagonal matrix such that $[\Lambda]=[\Pi]^{T}$. $[\Pi]$. This matrix enables to truncate the test functions such that $\mathbf{u} \in \mathcal{V}_{\Pi}$ is equal to zero over $\bar{\Omega}_{\Pi}^{0}$. The component $\Pi_{i j}$ is equal to zero for any degree of freedom $j$ located in $\bar{\Omega}_{\Pi}^{0}$, else it is equal to one. The number of rows of $[\Pi]$ is the number of degrees of freedom outside $\bar{\Omega}_{\Pi}^{0}$. Therefore $\Lambda_{j j}=1$ for any degree of freedom outside $\bar{\Omega}_{\Pi}^{0}$ else $\Lambda_{j j}=0$. Replacing the space of test function $\mathcal{V}_{h}$ by $\mathcal{V}_{\Pi}$ and $\mathcal{U}$ by $\mathcal{U}_{h}$ in Equation [7] we obtain a selection of equilibrium conditions:

$$
[\Pi] \cdot\{R\}\left(\{q\}, t,\{p\}_{\alpha}\right)=0
$$

The amount of equilibrium conditions related to the surrogate model has to be equal to the number of reduced state variables related to dispacements. Introducing the subspace of truncated test functions of the reduced-order model, $\mathcal{V}_{\Pi A}$, enables to obtain the convenient number of equilibrium condition.

$$
\begin{aligned}
& \mathcal{V}_{\Pi A}=\operatorname{span}\left\{\boldsymbol{\zeta}_{1}, \ldots, \boldsymbol{\zeta}_{\widehat{m}}\right\} \\
& \text { with } \quad \boldsymbol{\zeta}_{k}(\mathbf{X})=\sum_{j=1}^{j=n} \mathbf{N}_{j}(\mathbf{X}) \Lambda_{j j} A_{j k} \quad \forall k=1, \ldots, \widehat{m}
\end{aligned}
$$

The proposed formulation of the hyper-reduced-order model is obtained by replacing $\mathcal{V}$ by $\mathcal{V}_{\Pi{ }_{A}}$ and $\mathcal{U}$ by $\mathcal{U}_{P O D}$ in Equation [7]. The truncated test functions 
$\left(\boldsymbol{\zeta}_{k}(\mathbf{X})\right)_{k=1, \ldots, \widehat{m}}$ being constant and equal to zero over $\bar{\Omega}_{\Pi}^{0}$ the equilibrium equation of the hyper-reduced-order model can be simplified as:

$$
\begin{array}{r}
\int_{\Omega_{\Pi}^{0}} \varepsilon\left(\mathbf{u}_{\Pi A}^{*}, \mathbf{u}_{H R O M}\right): \boldsymbol{\Sigma}\left(\mathbf{F}\left(\mathbf{u}_{H R O M \tau}\right), \tau \leq t,\{p\}_{\alpha}\right) d \Omega^{0} \\
-\int_{\partial_{f} \Omega_{\Pi}^{0}} \mathbf{u}_{\Pi A}^{*} \cdot \mathbf{f}\left(\mathbf{X}, t,\{p\}_{\alpha}\right) d \Gamma^{0}=0 \\
\forall \mathbf{u}_{\Pi A}^{*} \in \mathcal{V}_{\Pi A} \text { with } \mathbf{u}_{H R O M} \in \mathcal{U}_{P O D}\left(\{p\}_{\alpha}\right)
\end{array}
$$

One can notice that $\mathbf{u}_{\Pi A} \in \mathcal{V}_{\Pi{ }_{A}}$ is equal to zero on the interface $\partial \bar{\Omega}_{\Pi}^{0} \cap \partial \Omega_{\Pi}^{0}$. This means that no interfacial equilibrium condition is checked in the proposed formulation. The boundary conditions over $\partial \bar{\Omega}_{\Pi}^{0} \cap \partial \Omega_{\Pi}^{0}$ is given by the shape of the POD-basis vector and the equilibrium conditions, Equation [46]. This formulation can be written in the following matrix form:

$$
[A]^{T} \cdot[\Lambda] \cdot\{R\}\left([A] \cdot\{a\}, t,\{p\}_{\alpha}\right)=0
$$

The linearized form of this problem is : find $\{\delta a\}$ such that,

$$
[A]^{T} \cdot[\Lambda] \cdot[K] \cdot[A] \cdot\{\delta a\}=-[A]^{T} \cdot[\Lambda] \cdot\{R\}\left([A] \cdot\{a\}, t,\{p\}_{\alpha}\right)
$$

where $[K]$ is the classical tangent stiffness matrix introduced for the Newton-Raphson algorithm. In case of differentiable governing equations this matrix is such that:

$$
K_{i j}=\frac{\partial R_{i}}{\partial q_{j}}
$$

To obtain a well-posed reduced problem, the number of selected equilibrium equations must be large enough. It can not be lower than the size of the reduced basis. Methodologies to build this selection of equations have been proposed in (Ryckelynck, 2005) and (Ryckelynck, 2009). In the present paper, we propose to use as a RID one of the subdomain of the domain decomposition in order to perform a simulation using a single processor.

Using the last formulation, we have reached the goal to reduce the complexity of the constitutive equations because the local computations are restricted to $\Omega_{\Pi}^{0}$. The HROM enables to forecast the reduced state variable $\{a\} .\{a\}$ being known, the displacement field is completely forecasted according to the reduced approximation in Equation [24]. The internal variables $\mathbf{z}$ are forecasted inside the RID in order to estimate $\boldsymbol{\Sigma}\left(\mathbf{F}\left(\mathbf{u}_{\tau}\right), \tau \leq t,\{p\}_{\alpha}\right)$. But they are not computed outside $\Omega_{\Pi}^{0}$. These internal variables can be extrapolated using an added POD basis as proposed in (Ryckelynck, 2009).

It is clear that $\mathbf{u}_{H R O M}$ and $\mathbf{u}_{P O D}$ can be different. The Hyper Reduction method introduces a new kind of approximation error. But, if the reference problem and 
the hyper-reduced problem have an unique solution, and if the finite element solution can be represented using a POD basis such that $\mathbf{u}_{h}=\mathbf{u}_{P O D}$, therefore the error introduced by the Hyper Reduction method is null. This is the reason why the added approximation error related to the Hyper Reduction method is viewed as an amplification of the error related to the POD representation. The proof of this property is straightforward. If $\mathbf{u}_{h}=\mathbf{u}_{P O D}$ then the residue of the equilibrium conditions $\{R\}\left([A] .\{a\}, t,\{p\}_{\alpha}\right)$ is null. Therefore the residue of the hyper-reduced problem is also null. So the column of reduced state variables $\{a\}$ is a solution of the hyper-reduced equations. Since this solution is unique, assuming that we can find it, there is no approximation error introduced by the hyper-reduction method: $\mathbf{u}_{h}=\mathbf{u}_{H R O M}$. For more details about the error amplification we refer the reader to (Ryckelynck, 2009).

The Hyper Reduction method remains unchanged the integration scheme over the RID. We do not propose a usual reduced integration scheme, but an efficient use of virtual work principle. Therefore we preserve the weight of each integration point and we preserve the mechanical sense of the internal variables. They remain the local variables defined by the Finite Element integration rule. In our point of view this is an important property. One can imagine to use one integration point per element as reduced integration scheme. But in such a case the mechanical sense of the internal variables has changed. It corresponds to average values over each element of the Finite Element model.

\section{A simple theoretical example}

We propose a simple mechanical example to facilitate numerical experiments of the Hyper reduction method. The series of problems involves 3 linear 1D tensil simulations. The displacement is such that: $\mathbf{u}=u\left(X,\{p\}_{\alpha}\right) \mathbf{e}_{1}$, where $\mathbf{e}_{1}$ is the first vector of the coordinate system (Figure 1). The displacement and the gradient of the displacement are assumed to be infinitely small $\left(\Omega=\Omega^{0}\right)$. The constitutive law is linear such that:

$$
\boldsymbol{\Sigma}\left(\mathbf{F}\left(\mathbf{u}_{\tau}\right), \tau \leq t,\{p\}_{\alpha}\right)=E\left(X,\{p\}_{\alpha}\right) \frac{\partial u}{\partial X} \mathbf{e}_{1} \otimes \mathbf{e}_{1}
$$

The Young modulus $E$ is a linear function of the position $X$. The unique parameter of the model is the value of the Young modulus at the end of the bar. The Young modulus at the origin is $1 \mathrm{Mpa}$. The 3 values of the parameter are $p_{1}=1 \mathrm{Mpa}, p_{2}=2 \mathrm{Mpa}$ and $p_{3}=4 \mathrm{Mpa}$. The displacement is imposed at the extremities of the bar (Figure 1). The mesh involves 9 linear elements. The length of the bar is 1 . There is 8 degrees of freedom supported by nodes 2 to 9 . The two first simulations provide two finite element displacement fields. By eliminating the given part of the displacement, we obtain two shape function $\boldsymbol{\psi}_{1}$ and $\boldsymbol{\psi}_{2}$ (Figure 2). In order to involve at least 2 finite element equilibrium conditions the RID can not be smaller than 3 elements in this case. We choose as the RID the last three elements of the mesh (Figure 1). We suggest the 


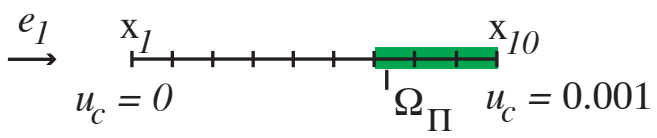

Figure 1. FE model, the RID covers the last three elements

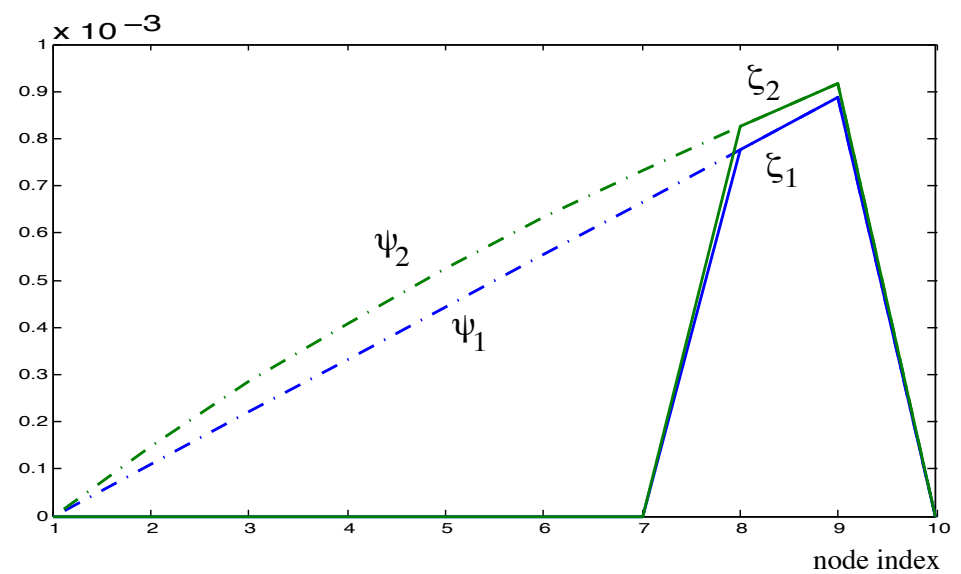

Figure 2. Shape functions and truncated test functions related to the hyper-reduced order model

reader to test various choice of RID. The two last degrees of freedom are outside the complementary part of the RID. Therefore, the matrix $[\Pi]$ is the following:

$$
[\Pi]=\left[\begin{array}{llllllll}
0 & 0 & 0 & 0 & 0 & 0 & 1 & 0 \\
0 & 0 & 0 & 0 & 0 & 0 & 0 & 1
\end{array}\right]
$$

The truncated test functions of the reduced order model $\zeta_{1}$ and $\zeta_{2}$ are shown in Figure 2.

The third simulation of the series has been performed three times using the full finite element model, a Galerkin formulation of the reduced approximation and the Hyper Reduced formulation. The related displacement fields are respectively denoted $u_{h}\left(x, p_{3}\right) u_{P O D}\left(x, p_{3}\right)$ and $u_{H R O M}\left(x, p_{3}\right)$. The relative errors $\frac{\left\|u_{P O D}-u_{h}\right\|}{\left\|u_{h}\right\|}$ and $\frac{\left\|u_{H R O M}-u_{h}\right\|}{\left\|u_{h}\right\|}$ are respectively 0.0107 and 0.0438. As mentioned above, the Hyper Reduction method gives rise to an error amplification. The bigger the RID the smaller this amplification is. The purpose of reduction methods is to reach a compromise between quality and the complexity of the surrogate model. Here, the reduced stiffness matrix is built using only three elements of the mesh. 


\section{Hyper-Reduction of a polycrystal plasticity model}

\subsection{Polycrystalline aggregate simulation details}

As a more complex example for the Hyper Reduction method application, a polycrystalline aggregate simulation was taken. This type of simulation is now classical in material science, either to deduce homogenized mechanical properties from the local microstructure knowledge (see for instance (Anand, 2004)) or to obtain local strain and stress fields (see for instance (Musienko et al., 2007) and references therein). For such a type of simulation, a finite element mesh of several hundreds of grains is typically used. Grain shapes can be simplified (cubes, octahedra), real-like (Voronoï polyhedra) or follow the real grain morhology. For each grain, a single crystal type of model is typically used, which assumes shear along appropriate slip systems. Such a simulation typically needs rather fine finite element mesh to properly capture the local heterogeneities. This naturally limits the possibilities for parametric investigations.

For the present work, we will use the possibilities of the Hyper Reduction method, in order to extend the studies of (Musienko et al., 2007) and check the boundary condition influence on the local stress-strain field.

\subsubsection{The constitutive model}

The constitutive model used here for each grain, (the same as in (Musienko et al., 2007)) was initially introduced in (Cailletaud, 1992). It was extensively used for the polycrystalline aggregate computations in a serie of works: (Barbe et al., 2001a)(Barbe et al., 2001b) - for the case of Ni-base alloy, (Diard et al., 2005) Zircaloy, (Osipov et al., 2008) - bainitic steel,(Gerard et al., 2009) - copper.

The formalism of the model, in a finite strain framework, is breathly recalled below. Mandel's isocline configuration (Mandel, 1973) is introduced, together with a multiplicative decomposition of the deformation gradient into an elastic and plastic part:

$$
\begin{aligned}
\mathbf{F} & =\mathbf{F}^{e} \mathbf{F}^{p} \\
\mathbf{S}^{e} & =\operatorname{det}\left(\mathbf{F}^{e}\right) \mathbf{F}^{e-1} \boldsymbol{\sigma} \mathbf{F}^{e-T} \\
\mathbf{T} & =\operatorname{det}(\mathbf{F}) \mathbf{F}^{e T} \boldsymbol{\sigma} \mathbf{F}^{e-T} \\
\rho^{o} & =\rho \operatorname{det}(\mathbf{F})
\end{aligned}
$$

where $\mathbf{T}$ is the Mandel tensor and $\mathbf{S}^{e}$ is the second Piola-Kirchhoff tensor related to the relaxed configuration. The elastic strain is expressed as:

$$
\mathbf{E}^{e}=\frac{1}{2}\left(\mathbf{F}^{e T} \mathbf{F}^{e}-\mathbf{I}\right)
$$

The model involves several slip systems. For each slip system $s$ an orientation tensor $\mathbf{N}^{s 0}$ describes the slip system geometry. The subscript "0" refers to the initial configuration. Each slip system $s$ involves kinematic hardening related to the internal variable 
$\alpha^{s}$. Isotropic hardenings are also introduced using the internal variables $v^{s}$. The set of internal variables is $\left\{\mathbf{E}^{e}, \alpha^{1}, \ldots, \alpha^{s}, \ldots, v^{1}, \ldots, v^{s}, \ldots\right\}$. It is corresponding to $\mathbf{z}$. The equation of state are:

$$
\mathbf{S}^{e}=\underset{\sim}{\mathbf{L} E^{e}} ; x^{s}=c \alpha^{s} ; r^{s}=R_{0}+b Q h_{s}\left(1-\exp \left(-b v^{s}\right)\right)
$$

The conjugate variables are $\left\{\mathbf{T}, x^{1}, \ldots, x^{s}, \ldots, r^{1}, \ldots, r^{s}\right\}$. The complementary constitutive equations are:

$$
\begin{aligned}
\dot{\mathbf{F}}^{p} \mathbf{F}^{p} & =\sum_{s} \dot{\gamma}^{s} \mathbf{N}^{s 0} \\
\dot{v}^{r} & =\left|\dot{\gamma}^{s}\right| \\
\dot{\alpha}^{s} & =\dot{\gamma}^{s}-d \dot{v}^{s} \alpha^{s} \\
\dot{\gamma}^{s} & =\left\langle\frac{\left|\tau^{s}-x^{s}\right|-r^{s}}{K}\right\rangle^{n} \operatorname{sign}\left(\tau^{s}-x^{s}\right) \\
\tau^{s} & =\mathbf{T}: \mathbf{N}^{s 0}
\end{aligned}
$$

\subsubsection{The material parameters}

The material parameters related to the constitutive law are: $\underset{\sim}{\mathbf{L}}-4$-order elasticity tensor, $R_{0}$ - yield limit, $Q$ and $b$ - nonlinear isotropic hardening parameters, $h_{s}$ - slip interaction matrix, $c$ and $d$ - nonlinear kinematic hardening parameters, $n$ and $K-$ viscosity parameters. The set of material parameters that taken from (Musienko et al., 2007) (even if it was recently modified in (Gerard et al., 2009)): $\mathrm{L}_{1111}=159,300 \mathrm{MPa}$; $\mathrm{L}_{1122}=121,900 \mathrm{MPa} ; \mathrm{L}_{1212}=80,900 \mathrm{MPa} ; \mathrm{K}=5 \mathrm{MPa} s^{1 / n} ; \mathrm{n}=10 ; \mathrm{c}=4,500 \mathrm{MPa}$; $\mathrm{d}=600 ; R_{0}=1.8 \mathrm{MPa} ; \mathrm{Q}=6 \mathrm{MPa} ; \mathrm{b}=15 ; \mathrm{h} 1=1.0 ; \mathrm{h} 2=4.4 ; \mathrm{h} 3=4.75 ; \mathrm{h} 4=4.75$; $\mathrm{h} 5=4.75 ; \mathrm{h} 6=5.0$.

\subsubsection{The finite element mesh}

The finite element mesh, used in (Musienko et al., 2007), was a numerical copy of about 100 grains of the copper specimen, representing a zone of 200x300x100 $\mu \mathrm{m}$, a part of 2x2x10 mm specimen. For the present work, we use a 200-grain finite element mesh (shown in the Figure 3), with the grains shaped as Voronoï polyhedra. Voronoï type of mesh is widely used to represent different materials, and thus such a choice will permit a more general interpretation of the results.

\subsubsection{Boundary conditions}

For the work of (Musienko et al., 2007), displacements were measured only at the surface of the specimen. That is why, an approximative solution was used for the boundary conditions. Namely, for all the surfaces, except one free surface, the displacements were prescribed:

$$
\mathbf{u}_{c}=\varepsilon \mathbf{x}
$$



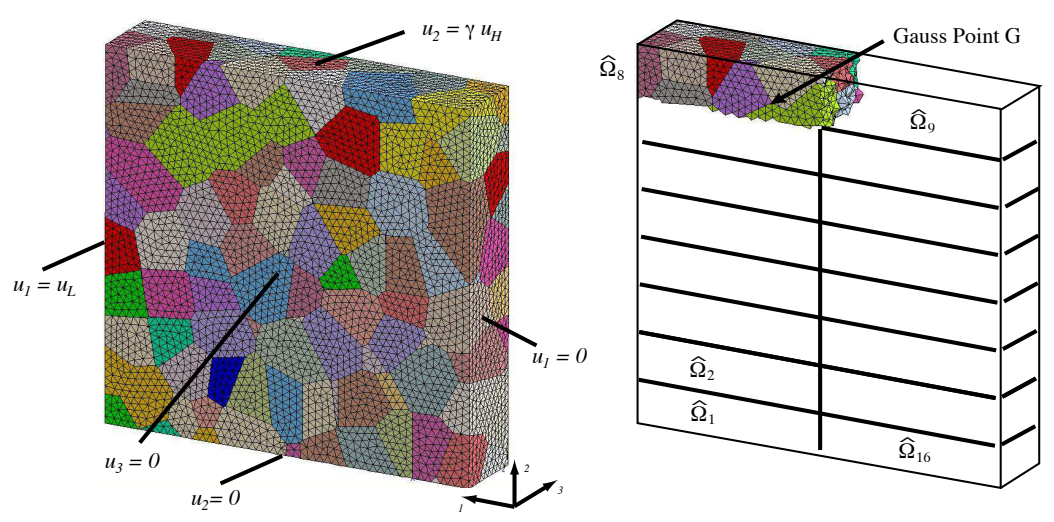

Figure 3. FE model, grains and domain decomposition

$$
\varepsilon=\left(\varepsilon_{11}, \varepsilon_{22}, \varepsilon_{33}\right)
$$

$\varepsilon_{11}$ was taken as the global strain of the specimen in axial direction. The transverse strains $\varepsilon_{22}$ and $\varepsilon_{33}$ were estimated through a simulation made for an elastoviscoplastic macroscopic model. For the present work, the displacement boundary conditions were kept. The displacement in tension direction (direction 1 in the Figure 3), was the same, as in (Musienko et al., 2007), i.e. $u_{1}=u_{L}=\varepsilon_{11} \cdot$ meshsize $_{1}$. The displacement in transverse direction (direction 2 in the Figure 3), was taken as $u_{2}=\gamma u_{H}=\gamma \varepsilon_{22}$. meshsize $_{2}$. Here $\gamma$ lies in the limits $[0.9,1$.$] .$

\subsection{Investigation of the state variation induced by modifications of a boundary condition}

The purpose of the series of simulations is to investigate the stress variation when changing the boundary condition $u_{2}=\gamma u_{H}$ on the top surface on Figure 3. $\gamma=1$ gives the boudary condition used in (Musienko et al., 2007). The series involves three simulations related to $\gamma=1, \gamma=0.9$ and $\gamma=0.95$. The third simulation aims to estimate if the variation of the stress field is a linear function of $\gamma$ for $\gamma$ in $[0.9,1$.$] .$

The two first simulations $(\gamma=1, \gamma=0.9)$ are performed using the full element model and a FETI solver (Feyel et al., 1997). The domain has been split into 16 subdomains. In order to show the usefulness of the Hyper Reduction method, the last simulation $(\gamma=0.95)$ has been done three times using the full finite element model, a POD-Galerkin formulation of the reduced approximation, Equation [39], and the Hyper Reduced formulation (Equation [46]). The related displacement fields are respectively denoted $\mathbf{u}_{h}\left(\mathbf{X}, t,\{p\}_{3}\right), \mathbf{u}_{P O D}\left(\mathbf{X}, t,\{p\}_{3}\right)$ and $\mathbf{u}_{H R O M}\left(\mathbf{X}, t,\{p\}_{3}\right)$. 


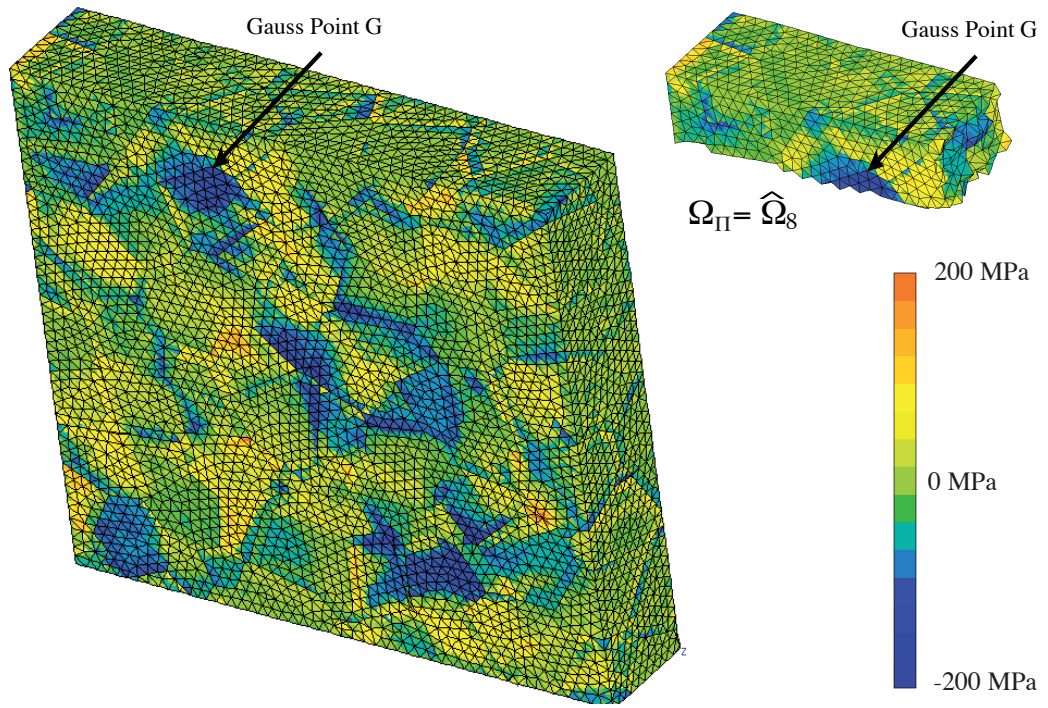

Figure 4. Stress $\sigma_{12}$ forecast by the FE model and the hyper-reduced model

The finite element model involves 869,274 degrees of freedom and 206,238 elements. Each simulation involves 29 time increments.

In order to save computational resources we choose a subdomain as the RID. To avoid the spurious estimate $\mathbf{u}_{H R O M}\left(\mathbf{X}, t,\{p\}_{3}\right)=0$ the RID must cover a part of the boundary where the displacement are prescribed such that $[\Pi] .\{R\}\left(0, t,\{p\}_{3}\right)$ is not equal to zero. Therefore, we have chosen $\Omega_{\Pi}^{0}=\widehat{\Omega}_{8}^{0}$ (Figure 3 ). The RID involves 12,187 elements.

The snapshot POD have been applied to the 58 continuous mechanical states provided at the end of each time increment of the two first simulations. It provided 5 POD basis vectors $\left(\boldsymbol{\psi}_{k}\right)_{k=1 \ldots \hat{m}=4}$. As shown in Figure 4 , the Cauchy stress $\sigma_{12}$, related to $\gamma=0.95$, forecast by the HROM, at the end of the time interval, is in good agreement with the one farecast by the Finite Element method and the FETI solver. The variation of the Cauchy stress is denoted $\delta \sigma_{12}=\sigma_{12 \mid \gamma=0.95}-\sigma_{12 \mid \gamma=1}$. The time evolution at $G$ point of the various estimates of $\delta \sigma_{12}$ is shown on Figure 5 .

In so far as some of the residues of the equilibrium equations are not included into the Hyper reduced formulation, this formulation introduces new approximation errors. It is well known that the Galerkin procedure allows to minimize the approximation errors when the equilibrium equations derived from an error minimization. Therefore, one can expect some error amplification due to the Petrov-Galerkin formulation used for the Hyper Reduction. One can notice that the HROM estimation of $\delta \sigma_{12}$ is very 

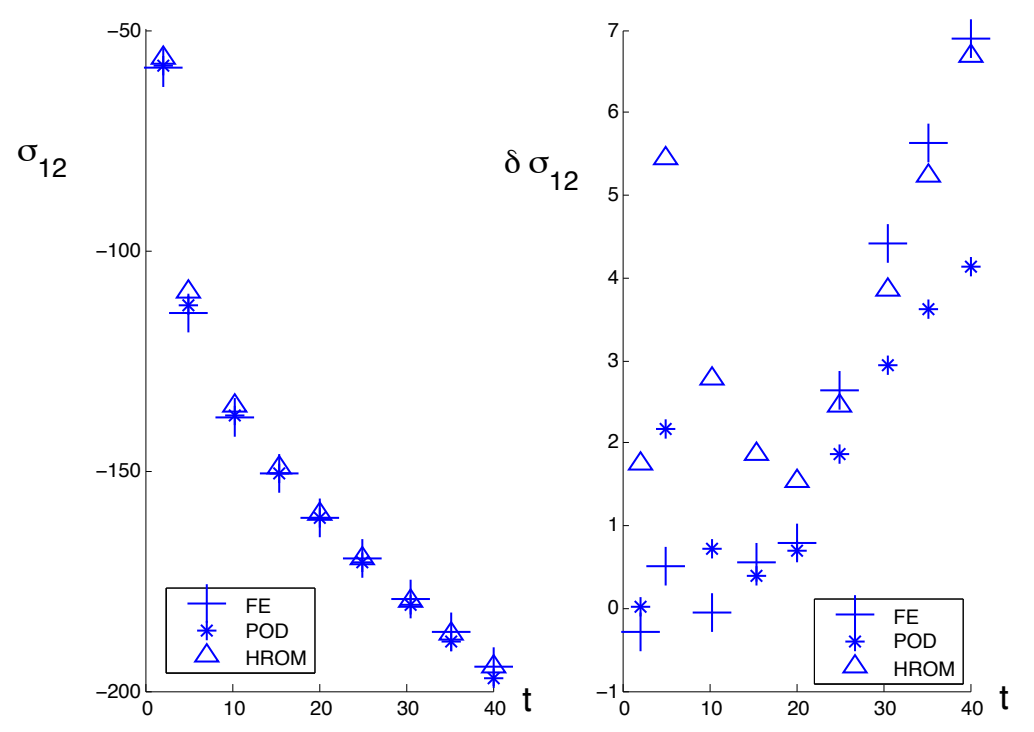

Figure 5. $\sigma_{12 \mid \gamma=0.95}$ and $\delta \sigma_{12}=\sigma_{12 \mid \gamma=0.95}-\sigma_{12 \mid \gamma=1}$ at G point (Figure 3)

accurate at the end of the time interval at $G$ point (Figure 3 and Figure 5). The error amplification due to the Hyper Reduced formulation has an impact at the beginning of the time interval, where the reduced approximation is less accurate.

Anyway, these results are accurate enougth to validate the putative linear variation of the stress when changing $\gamma$ in the interval $[0.9,1]$. The smoother the state variation when changing $\gamma$, the bigger should be the validity domain of the Hyper Reduced approximation. The norm of truncated residual of the reference equilibrium equations should be helpfull to estimate a trust region. The relative error on truncated equilibrium equation $\eta_{R}$ and the true relative error on $\sigma_{12}$ at point $G$, defined below, are shown on Figure 6.

$$
\begin{aligned}
& \eta_{R}=\max _{t} \frac{\|[\Pi] \cdot\{R\}([A] \cdot\{a\}, t, \gamma=0.95)\|}{\|[\Pi] \cdot\{R\}(0, t, \gamma=0.95)\|} \\
& \eta_{\sigma}=\max _{t} \frac{\left|\sigma_{12 \text { H } R O M}\right| X=G, \gamma=0.95-\sigma_{12} h|X=G, \gamma=0.95|}{\left|\sigma_{12} h\right| X=G, \gamma=0.95 \mid}
\end{aligned}
$$



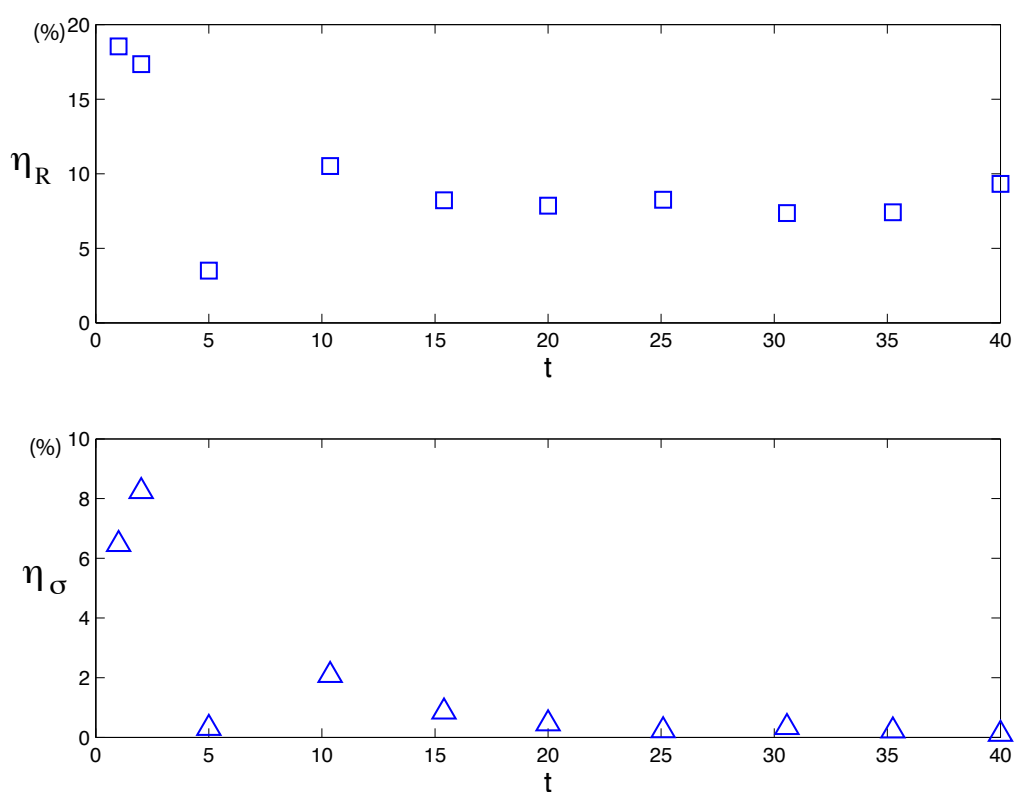

Figure 6. $\sigma_{12 \mid \gamma=0.95}$ and $\delta \sigma_{12}=\sigma_{12 \mid \gamma=0.95}-\sigma_{12 \mid \gamma=1}$ at $G$ point

\subsection{Discussion on the energy consumption during the last simulation}

Each simulation related to $\gamma=0.95$ involves 29 time increments. The FE simulation involves 4 Newton-Raphson iterations per time increment, and 180 FETI iterations per linear global system to solve. The duration of the simulation is given by the slowest processor in order to perform the computation. This time duration was $420,067 \mathrm{~s}$. The reduced approximations being continuous overall the domain, a direct solution of the linear global equations (Equation [47]) is performed using a classical Gauss solver. The Hyper reduced simulation involves 5 Newton-Raphson iterations per time increment, and one solution of the linear global system (Equation [47]) for each iteration. This simulation was performed on only one processor. The time duration of this simulation was $1,165 \mathrm{~s}$. The duration of the POD-Galerkin simulation, was $2,053 \mathrm{~s}$.

The Hyper Reduction method provides an amazing Computational Time Saving Factor (CTSF) equal to 361. The POD simulation is less efficient than the HROM one because of the assembly procedure of the tangent stiffness matrix of the global linear systems embeded in the Newton-Raphson algorithm. But the most important result of this work is the capability of the Hyper Reduction method to reduce the computational resource needed to perform the simulation. The processor used to perform the HROM simulation has the following characteristics: $2 \mathrm{Ghz}, 4 \mathrm{Go}$ for memory. Such processor 
can be found on a classical laptop. The use of a single processor during less time enables to save electrical energy during the numerical simulation. An estimated energy saving factor $s_{E}$ enables to quantify the resource reduction such that:

$$
s_{E}=\frac{\omega_{F E}}{\omega_{H R O M}} C T F S
$$

The estimated energy saving factor of the Hyper Reduction method is 5,769. It is 28 times bigger than the one provided by the classical POD-Galerkin procedure which use all the processors related to the domain decomposition.

\section{Conclusion}

In continuation of previous work related to three-dimensional finite element simulation of a polycrystalline copper specimen using experimentally determined microstructure, we investigate the stress variation related to model modifications. A POD approximation has been build to reproduce the displacements forecast using two finite element simulations related to different parameter values. This POD approximaton has been used to forecast the mechanical response related to an other parameter value. The reduced state variables have been computed using the Hyper Reduction method. Despite the full finite element simulations requires 16 processors of a cluster and the FETI method, the reduced state variables have been forecast using only one processor. This approch is twice more efficient than the classical POD-Galerkin procedure. Compared to the Finite Element method, an amazing saving factor related to computational time has been reached. This factor is 361. But much more interesting results are related to the ressource saving provided by the Hyper Reduction method. An energy saving factor has been proposed estimating the energy saving related to a simulation. This saving factor is the saving factor related to the computational time multiplied by the ratio of processor used to perform the simulation. For the proposed example this ratio is 16 . Then we achieve an estimated energy saving factor equal to 5,769. At last, we must emphasis that the Hyper Reduced simulation can be performed using a classical laptop instead of a parallel computer. Most of the parallel computers are optimized to maximize the availability of their processors for computational purposes. On the contrary, the laptop computer processors are designed to minimize their energy consumption. It turns out that, replacing some simulations on parallel computer by simulations on laptops, the Hyper Reduction method enables a first step toward "green" mechanical simulations in materials science.

Works in process concern the extent of the applicability of the Hyper Reduction method. This approche seems interesting to investigate the variation of the forecast state variables depending on variation of parameters of the model. It could be parameters related to constitutive equations or loading conditions. 


\section{Acknowledgements}

This work is related to the MELOXEL BLAN07-2-18671 project partially funded by the French National Research Agency (ANR).

\section{References}

Anand L., " Single-crystal elasto-viscoplasticity: application to texture evolution in polycrystalline metals at large strains", Computer Methods in Applied Mechanics and Engineering, vol. 193, n 48-51, p. 5359-5383, 2004.

Balima O., Favennec Y., Girault M., Petit D., " Comparison between the modal identification method and the POD-Galerkin method for model reduction in nonlinear diffusive systems", International Journal for Numerical Methods in Engineering, vol. 67, $\mathrm{n}^{\circ}$ 7, p. 895-915, 2006.

Barbe F., Decker L., Jeulin D., Cailletaud G., " Intergranular and intragranular behavior of polycrystalline aggregates Part 1: model”, Int. J. of Plasticity, vol. 17, $\mathrm{n}^{\circ}$ 4, p. 513-536, $2001 \mathrm{a}$

Barbe F., Forest S., Cailletaud G., “ Intergranular and intragranular behavior of polycrystalline aggregates.Part 2: Results”, Int. J. of Plasticity, vol. 17, n 4, p. 537-563, 2001b.

Cailletaud G., " A micromechanical approach to inelastic behaviour of metals”, Int J of Plasticity, vol. 8, n 1, p. 55Đ73, 1992.

Craig R. J., Bampton M., “ Coupling of substructures for dynamic analyses”, AIAA Journal, vol. 6, n 7, p. 1313Đ1319, 1968.

Daescu D. N., Navon I. M., " Efficiency of a POD-based reduced second-order adjoint model in 4D-Var data assimilation", International Journal for Numerical Methods in Fluids, vol. $53, n^{\circ} 6$, p. 985-1004, 2007.

Diard O., Leclercq S., Rousselier G., Cailletaud G., "Evaluation of finite element based analysis of 3D multicrystalline aggregates plasticity: Application to crystal plasticity model identification and the study of stress and strain fields near grain boundaries", Int. J. of Plasticity, vol. 21, n 4, p. 691-722, 2005.

Farhat C., Roux F.-X., “ Implicit parallel processing in structural mechanics”, Computational Mechanics Advances, vol. 2, p. 1-124, 1994.

Feyel F., Calloch S., Marquis D., Cailletaud G., " F.E. Computation of a Triaxial Specimen Using a Polycrystalline Model”, CMS, vol. 9, p. 141-157, 1997.

Forest S., Pilvin P., "Modelling finite deformation of polycrystals using local objective frames”, Z. Angew. Math. Mech., vol. 79, p. S199-S202, 1999.

Ganapathysubramanian S., Zabaras N., " Design across length scales: a reduced-order model of polycrystal plasticity for the control of microstructure-sensitive material properties", Comput. Methods Appl. Mech. Engrg., vol. 193, p. 5017-5034, 2004.

Gerard C., Nguyen F., Osipov N., Cailletaud G., Bornert M., " Comparison of experimental results and finite element simulations of strain localization scheme under cyclic loading path”, Computational Materials Science, vol. 46, p. 755-760, 2009.

Germain P., Nguyen Q. S., Suquet P., “ Continuum Thermodynamics”, Journal of Applied Mechanics, vol. 50, p. 1010-1020, 1983. 
Holmes P., Lumley J.-L., G B., Turbulence, Coherent Structures, Dynamical Systems and Symetry, 1st edn, Cambridge University Press, 1998.

Karhunen K., " Uber lineare methoden in der wahrscheinlichkeitsrechnung”, Ann. Acad. Sci. Fennicae, ser. Al. Math. Phys., 1946.

Khalila M., Adhikarib S., Sarkara A., “ Linear system identification using proper orthogonal decomposition”, Mechanical Systems and Signal Processing, vol. 21, p. 3123Đ3145, 2007.

Ladevèze P., “ Sur une famille d'algorithmes en mécanique des structures”, Comptes Rendus Acad. Sci. Paris Sèrie II, vol. 300, n² 2, p. 41-44, 1985.

Lemaitre J., Chaboche J.-L., Mecanique des materiaux solides, 1st edn, Dunod: Paris 1985, English version published by Cambridge University Press: cambridge, 1990.

Lieu T., Farhat C., Lesoinne M., " Reduced-order fluid/structure modeling of a complete aircraft configuration", Computer Methods in Applied Mechanics and Engineering, vol. 95, $\mathrm{n}^{\circ} 41-$ 43, p. 5730-5742, 2006.

Lorenz E. N., Empirical Orthogonal Functions and Statistical Weather Prediction, Scientific Report $n^{\circ} 1$, MIT Departement of Meteorology, Statistical Forecasting Project, 1956.

Loève M. M., Probability theory, The University Series in Higher Mathematics, 3rd edn, Van Nostrand, Princeton, NJ, 1963.

Mandel J., "Equations constitutives et directeurs dans les milieux plastiques et viscoplastiques", Int J Solids Structures, vol. 9, n 6, p. 725Đ40, 1973.

Musienko A., Tatschl A., Schmidegg K., Kolednik O., Pippan R., Cailletaud G., " Threedimensional finite element simulation of a polycrystalline copper specimen", Acta Materialia, vol. 55, p. 4121-4136, 2007.

Osipov N., Gourgues-Lorenzon A.-F., Marini B., Mounoury V., Nguyen F., Cailletaud G., “ FE modelling of bainitic steels using crystal plasticity”, Philosophical Magazine, vol. 88, n 3032, p. 3757-3777, 2008.

Ryckelynck D., “A priori hypereduction method: an adaptive approach”, International Journal of Computational Physics, vol. 202, p. 346-366, 2005.

Ryckelynck D., “ Hyper reduction of mechanical models involving internal variables”, International Journal for Numerical Methods in Engineering, vol. 77, n 1, p. 75-89, 2009.

Ryckelynck D., Benziane D.-M., “ Multi-level a priori hyper-reduction of mechanical models involving internal variables", Computer Methods in Applied Mechanics and Engineering, vol. 199, n¹7-20, p. 1134-1142, 2010.

Sansour C., Kollmann F., “ On theory and numerics of large viscoplastic deformation”, Comput. Methods Appli. Mech. Engrg., vol. 146, p. 351-369, 1997.

Sirovich L., " Turbulence and the dynamics of coherent structures partI : coherent structures", Quaterly of applied mathematics, vol. XLV, n 3, p. 561-571, 1987.

Tugcu P., Neale K. W., Wu P. D., Inal K., “ Crystal plasticity simulation of the hydrostatic bulge test”, Int. Jour. of Plasticity, vol. 20, p. 1603-1653, 2004.

Zienkiewicz O., Taylor RL. Finite Element Method, vol. 1-3, Butterworth-Heinomann, London, 2000 .

Received: 4 November 2009

Accepted: 12 March 2010 